EPJ Web of Conferences 36, 00007 (2012)

DOI: $10.1051 /$ epjconf/20123600007

CC Owned by the authors, published by EDP Sciences, 2012

\title{
Resonances production from the NA60 experiment
}

\author{
A. De Falco ${ }^{1, a}$ for the NA60 Collaboration \\ ${ }^{1}$ Università and INFN Cagliari
}

\begin{abstract}
The NA60 experiment at the CERN SPS has studied light vector meson production in In-In collisions at $158 \mathrm{~A} \mathrm{GeV}$. The $\phi$ meson was detected via both the $K^{+} K^{-}$ and the $\mu^{+} \mu^{-}$decay channels. The yields and inverse slope parameters of the $m_{T}$ spectra observed in the two channels are compatible within errors, different from the large discrepancies seen in $\mathrm{Pb}-\mathrm{Pb}$ collisions between the hadronic (NA49) and dimuon (NA50) decay channels. In the invariant mass region $0.2<M_{\mu \mu}<2.6 \mathrm{GeV}$, a strong excess of pairs above the sources that describe the mass spectrum in p-A collisions is observed. The mass spectrum for $M_{\mu \mu}<1 \mathrm{GeV}$ is consistent with a dominant contribution from the pion annihilation process $\pi+\pi \rightarrow \rho \rightarrow \mu^{+} \mu^{-}$. For $M_{\mu \mu}>1 \mathrm{GeV}$, the excess is found to be prompt, with remarkable differences with respect to the Drell-Yan process. The $T_{\text {eff }}$ slope parameter, extracted from the transverse mass spectra, shows a rise with mass up to the $\rho$, followed by a sudden decline for higher masses. The former is consistent with radial flow of a hadronic source, while the latter suggests a dominantly partonic emission source.
\end{abstract}

\section{Introduction}

The theory of Quantum Chromo-Dynamics (QCD) predicts that matter under extreme conditions of temperature and energy density undergoes a phase transition from hadronic matter to a plasma of deconfined quarks and gluons (QGP). The occurrence of this phase can be studied in the laboratory by means of high energy heavy-ion collisions. Dileptons can be used to detect vector mesons, and are particularly attractive to study the QGP phase since, differently from hadrons, they are not perturbed by final-state interactions. In this paper, results on $\phi$ production and on the $\rho$ spectral function in In-In collisions are reported.

The $\phi$ meson, being composed of an $s \bar{s}$ pair, is an ideal probe for the study of strangeness production, which has been suggested as a probe of QGP formation [1]. Moreover, the $\phi$ mass and branching ratios for the decay into kaon and lepton pairs may be modified in the medium $[2,3]$.

$\phi$ meson production was formerly studied at the CERN SPS by the NA49 [4,5] and NA50 [6,7] experiments, that detected the $\phi$ through its decay into $K^{+} K^{-}$and $\mu^{+} \mu^{-}$pairs, respectively. The NA49 measurement is dominated by low transverse momenta, and is limited by statistics to $p_{T} \lesssim 1.6 \mathrm{GeV} / c$. On the contrary, the NA50 acceptance is limited to $p_{T}>1.1 \mathrm{GeV} / c$.

The results obtained by NA50 and NA49 in Pb-Pb collisions show discrepancies both in the yield and in the inverse slope parameter $T_{\text {eff }}$ of the $m_{T}$ spectra. The $\phi$ multiplicity in central $\mathrm{Pb}-\mathrm{Pb}$ collisions measured by NA50 in the dimuon channel is higher by about a factor of 4 with respect to the corresponding NA49 measurement in the $K^{+} K^{-}$channel. The $T_{\text {eff }}$ value found by NA50 is about 220 $230 \mathrm{MeV}$, showing a mild dependence on centrality, while NA49 measured an inverse $m_{T}$ slope that increases with centrality and saturates at $T_{\text {eff }} \sim 300 \mathrm{MeV}$.

The discrepancy between the NA49 and NA50 results, known as " $\phi$ puzzle", triggered a considerable theoretical effort to explain the observed differences [8-11]. It was argued that in-medium effects may affect the spectral function of the $\phi$, causing a modification of its mass and partial decay widths. Moreover, kaon absorption and rescattering in the medium can result in a loss of signal in the region

\footnotetext{
${ }^{a}$ e-mail: alessandro.de.falco@ca.infn.it
}

This is an Open Access article distributed under the terms of the Creative Commons Attribution License 2.0, which permits unrestricted use, distribution, and reproduction in any medium, provided the original work is properly cited. 
of the $\phi$ invariant mass in the $K^{+} K^{-}$channel, thus reducing the observed yield. This effect would be concentrated at low $p_{T}$, causing a hardening of the $p_{T}$ spectrum in this channel $[11,9]$. Nevertheless, according to those calculations, the yield in lepton pairs is expected to exceed the one in kaon pairs by about $50 \%$, which is much lower than the observed differences.

Subsequent measurements by the CERES [12] Collaboration in $\mathrm{Pb}-\mathrm{Au}$ collisions added further information about $\phi$ production at the SPS. CERES could access both the $K^{+} K^{-}$and $e^{+} e^{-}$channels, the former in the region $p_{T}>0.75 \mathrm{GeV} / c$, the latter for $p_{T}<1.5 \mathrm{GeV} / c$ due to limitations in statistics. The CERES measurement in both channels is in agreement with the NA49 measurement. However, CERES' measurement of the $T_{\text {eff }}$ parameter in the dileptonic channel is affected by a large statistical uncertainty that does not allow to draw firm conclusions. Thanks to its muon spectrometer and vertex tracker, that will be described below, NA60 could access both the $\mu^{+} \mu^{-}$[13] and $K^{+} K^{-}$[14] channels, and thus study the possible existence of a " $\phi$ puzzle" in In-In collisions.

The $\rho$ meson is particularly important in the study of the hot and dense state formed in high-energy heavy ion collisions, due to its strong coupling to the $\pi \pi$ channel and the short lifetime $(\sim 1.3 \mathrm{fm} / c)$, which is much shorter than the lifetime of the fireball. Due to these characteristics, its in-medium properties, like mass and width, have long been considered as an ideal signature for the restoration of chiral symmetry [15-17], which is associated to the phase transition between hadronic matter and the QGP. At intermediate masses (IMR, $M>1 \mathrm{GeV} / c^{2}$ ), Planck-like thermal radiation is expected from both partonic and hadronic sources. The measurement of dilepton mass and transverse momentum distributions should allow to differentiate between the two. Previous measurements by CERES [18, 19] for $M<1 \mathrm{GeV} / c^{2}$, NA38/50 [20] for $M>1 \mathrm{GeV} / c^{2}$, and HELIOS-3 [21] in both regions, showed an excess of the data with respect to the expected sources that led to a strong theoretical effort. At low masses (LMR, $M<1 \mathrm{GeV}$ ), $\pi \pi$ annihilation, with regeneration and in-medium modifications of the $\rho$ meson during the fireball expansion were identified as the dominant source of this excess. However, the limited statistics and mass resolution were not sufficient to give a precise assessment on the properties of the $\rho$. The NA60 experiment, with its high statistics, high resolution measurement, allowed to clarify the open issues left in the previous measurements [22-25]. In this paper, the most important results on the excess dimuons detected by NA60 are summarized.

\section{Experimental apparatus}

The detectors relevant for the present work are a muon spectrometer (MS) inherited from NA50 and a vertex telescope (VT). They are described in detail in [26,27]. The muon spectrometer is composed of a toroidal air-core magnet (ACM), 8 multi-wire proportional chambers for the tracking and a set of scintillator hodoscopes that provides the main trigger, which selects muon pairs coming from a common vertex. The muon trigger does not introduce any selection in the dimuon mass and $p_{T}$. The selectivity of the trigger allows to run at high beam intensities. The angular acceptance is $35<\theta<120$ mrad; the rapidity coverage for the $\phi$ is $3 \lesssim y \lesssim 4.2$. The muon spectrometer is preceded by a $12 \lambda_{I}$ thick hadron absorber.

The VT is a high-granularity, radiation tolerant Si pixel detector, placed between the target and the absorber in a $2.5 \mathrm{~T}$ dipole field. It is used to determine the primary vertex, the charged particle multiplicity and to measure the momentum of the charged tracks. The telescope used during the indium run consisted of 16 independent detector planes, providing 11 tracking points, arranged along the beam axis over a length of $\sim 26 \mathrm{~cm}$, starting at $\sim 7 \mathrm{~cm}$ from the target centre. The telescope covers the angular acceptance of the muon spectrometer.

\section{Data sample and analysis}

During the 5 -week-long run in 2003 , about $3 \cdot 10^{12}$ ion beams were delivered to the experiment. 230 million muon pair triggers were recorded, with two different settings of the ACM magnet current (4000 A and $6500 \mathrm{~A}$ ). The results presented in this paper refer to the $4000 \mathrm{~A}$ data sample, which 

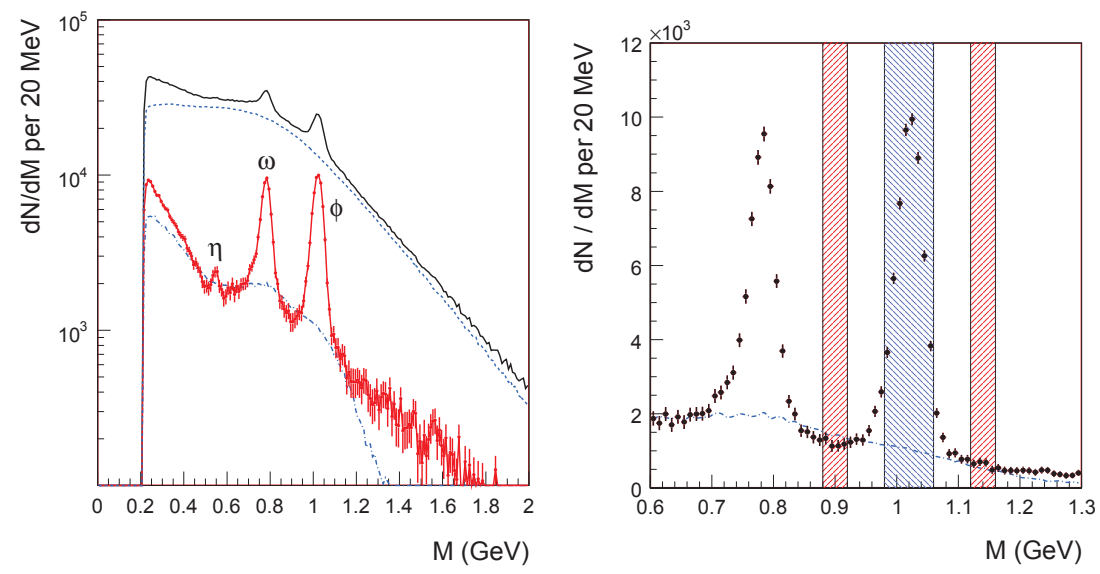

Fig. 1. Left panel: dimuon raw mass distribution (continuous line), combinatorial background (dashed line), fake tracks (dot-dashed line) and mass distribution after combinatorial background and fake tracks subtraction (continuous curve with error bars). Right panel: mass region around the $\phi$ peak and mass windows used in the analysis after backgrounds subtraction. The dot-dashed distribution is the total fake matches distribution.

amounts to $\sim 50 \%$ of the total statistics, as the 6500 A setting suppresses the low mass acceptance and it is optimal for the study of the $J / \psi$.

The data were collected with a $158 \mathrm{~A} \mathrm{GeV}$ indium beam, incident on 7 indium targets of $17 \%$ total interaction probability. The targets were placed in vacuum in order not to pollute the data sample with In-air interactions. The primary vertex was reconstructed with a precision of $10-20 \mu \mathrm{m}$ in the transverse coordinates and $\sim 200 \mu \mathrm{m}$ in the longitudinal coordinate. The target identification is extremely good, with negligible background between different targets.

Muon tracks are first reconstructed in the muon spectrometer. They are then extrapolated back to the target region and matched to the tracks reconstructed in the vertex spectrometer. This is done comparing both their angles and momenta, requiring a matching $\chi^{2}$ less than 3 . Once identified, the muons are refitted using the joint information of the muon and the vertex spectrometers. Muon pairs of opposite charge are then selected. The matching technique drastically improves the signal-to-backround ratio and the dimuon mass resolution, which improves from $\sim 80 \mathrm{MeV}$ (using only the information from the muon spectrometer) down to $\sim 20 \mathrm{MeV}$ at the $\phi$ peak, independent of centrality [22].

Events with more than one interaction vertex or with a (reconstructed) charged track multiplicity smaller than 4 are discarded.

The matched dimuon mass spectrum is shown in the left panel of Fig. 1. The same picture shows also the two background sources: combinatorial and fake matches. The former is made of uncorrelated muon pairs, mainly coming from weak decays of pions and kaons in the same event, and is evaluated through an event mixing technique, with an accurancy of $\sim 1 \%$ over the full dimuon mass spectrum. The latter is due to an incorrect matching between the tracks reconstructed in the MS and in the VT. It is estimated either with an overlay Monte Carlo simulation, where a simulated dimuon is reconstructed on top of a real event, or mixing the MS muons of a given event with the VT tracks of another event. The two methods agree within 5\%.

As it can be seen, the dominant background is the combinatorial background. The $\omega$ and $\phi$ peaks are very well resolved and the $\eta$ peak is also visible. After the subtraction of combinatorial background and fake matches, the dimuon invariant mass spectrum contains about 360000 events in the mass range $2 m_{\mu \mu}<M<2 \mathrm{GeV} / c^{2}$. 


\section{$4 \phi$ production}

\subsection{Analysis in the dimuon channel}

To study $\phi$ production, events in a small mass window centred at the $\phi$ pole mass $(0.98<M<$ $1.06 \mathrm{GeV} / c^{2}$ ) are selected from the mass distribution after the combinatorial background subtraction. The right panel of Fig. 1 shows an expanded view of the $\omega$ and $\phi$ region after the subtraction of background. The sample consists of $\sim 70000 \phi$ events. The signal/background ratio, integrated over centrality, is $\sim 1 / 3$ below the $\phi$ peak, ranging from about 3 in peripheral events to about $1 / 5$ in central ones.

The continuum below the $\phi$ is accounted for by subtracting the events in two side mass windows shown in the right panel of Fig. $1\left(0.88<M<0.92 \mathrm{GeV} / c^{2}\right.$ and $\left.1.12<M<1.16 \mathrm{GeV} / c^{2}\right)$.

The number of $\phi$ mesons in the muon channel is measured counting the events in the mass window $0.98<M_{\mu \mu}<1.06 \mathrm{GeV} / c^{2}$. The signal/background ratio below the $\phi$ peak, integrated over centrality, is $\sim 1 / 3$. To account for the continuum under the $\phi$ peak, two side windows between $0.88<M_{\mu \mu}<$ $0.92 \mathrm{GeV} / c^{2}$ and $1.12<M_{\mu \mu}<1.16 \mathrm{GeV} / c^{2}$ are subtracted from the $\phi$ window. The width and position of the side bands are varied and the differences in the results are included in the systematic uncertainty. The raw transverse momentum distributions are extracted counting the number of $\phi$ mesons in several $p_{T}$ intervals. They are then corrected for the acceptance using an overlay Monte Carlo tuned iteratively such that the resulting distributions reproduce the measured data [28]. The systematic error is evaluated by varying the analysis parameters and selections. The measurement is performed for 5 centrality bins, corresponding to $\left\langle N_{\text {part }}\right\rangle=15,41,78,133,177$.

The $\phi$ multiplicity is estimated either with a direct measurement of the cross section, or using the $\mathrm{J} / \psi$, corrected for nuclear and anomalous absorption, as a calibration process [28]. The two methods agree within $10 \%$.

\subsection{Analysis in the kaon channel}

The analysis in the $K^{+} K^{-}$channel is performed assuming that all the charged particles associated with the primary vertex are kaons, and building all the possible opposite sign pairs among the tracks of each event. The high resulting combinatorial background is reduced applying cuts on the pair's opening angle $\left(0.005<\theta_{K K}<0.15 \mathrm{rad}\right)$ and transverse momentum $\left(p_{T}>0.9 \mathrm{GeV} / c\right)$, while a selection in the rapidity region of the track $2.9<y_{K}<3.7$ excludes the borders of the detector acceptance. After applying these cuts, the ratio between background and signal ranges from $\sim 190$ to $\sim 460$, depending on centrality.

The residual background is subtracted with an event mixing technique. Mixed events are subject to the same cuts as the ones applied to the real data. The obtained mixed spectra are normalized such that, for like-sign pairs, the integrals of the real and mixed spectra coincide in the mass window $1.02<M<$ $1.06 \mathrm{GeV} / c^{2}$.

The invariant mass spectrum obtained after background subtraction is displayed in Fig. 2, integrated in centrality. The mass spectrum still contains unsubtracted combinatorial background, which is described in the fit by an empirical polynomial function. Several functions are used to describe the residual background, including polynomial of. Differences in the results due to the choice of the fit function contribute to the systematic uncertainty. More details are reported in [14]. For the $\phi$ peak, a parametrization obtained from a fit to the mass distribution obtained from an overlay simulation of the signal is used. When the $\phi$ peak position and width are left as free parameters, the corresponding values are $m_{\phi}=1019.5 \pm 0.3 \pm 1.2 \mathrm{MeV} / c^{2}$ and $\sigma_{m}=7.8 \pm 0.3 \pm 1.2 \mathrm{MeV} / c^{2}$, in good agreement with the simulations. No centrality dependence is observed for these values.

The $m_{T}$ distributions are extracted fitting the invariant mass spectra in several $m_{T}$ intervals, and correcting the results for the acceptance and efficiency, obtained with an overlay Monte Carlo simulation. The acceptance multiplied by the reconstruction efficiency for $p_{T}>0.9 \mathrm{GeV} / c$ is about $15 \%$ in full rapidity, and almost flat as a function of $p_{T}$ (more details are reported in [14]). Due to the limited statistics transverse mass distributions are extracted for central and semi-central collisions only $\left(\left\langle N_{\text {part }}\right\rangle=177\right.$ and 133, respectively). 
Table 1. $\phi$ inverse slope and yield extracted from the analyses in the muon and kaon channels as a function of centrality.

\begin{tabular}{cccccc}
\hline$\left\langle N_{\text {part }}\right\rangle$ & $T_{\text {eff }}^{\mu \mu}(\mathrm{MeV})$ & $\langle\phi\rangle_{\mu \mu}\left(p_{T}>0.9 \mathrm{GeV}\right)$ & $T_{\mathrm{eff}}^{K K}(\mathrm{MeV})$ & $\langle\phi\rangle_{K K}\left(p_{T}>0.9 \mathrm{GeV}\right)$ & $\langle\phi\rangle_{K K}\left(p_{T}>0\right)$ \\
\hline 15 & $209 \pm 4$ & $0.044 \pm 0.002 \pm 0.005$ & - & - & - \\
41 & $232 \pm 4$ & $0.197 \pm 0.009 \pm 0.021$ & - & $0.16 \pm 0.05 \pm 0.08$ & $0.64 \pm 0.17 \pm 0.03$ \\
78 & $245 \pm 4$ & $0.48 \pm 0.02 \pm 0.05$ & - & $0.47 \pm 0.06 \pm 0.02$ & $1.60 \pm 0.18 \pm 0.06$ \\
133 & $250 \pm 4$ & $1.03 \pm 0.03 \pm 0.07$ & $253 \pm 11 \pm 5$ & $1.01 \pm 0.07 \pm 0.05$ & $3.5 \pm 0.2 \pm 0.2$ \\
177 & $249 \pm 5$ & $1.65 \pm 0.06 \pm 0.07$ & $254 \pm 13 \pm 6$ & $1.49 \pm 0.11 \pm 0.07$ & $4.6 \pm 0.3 \pm 0.4$ \\
\hline
\end{tabular}
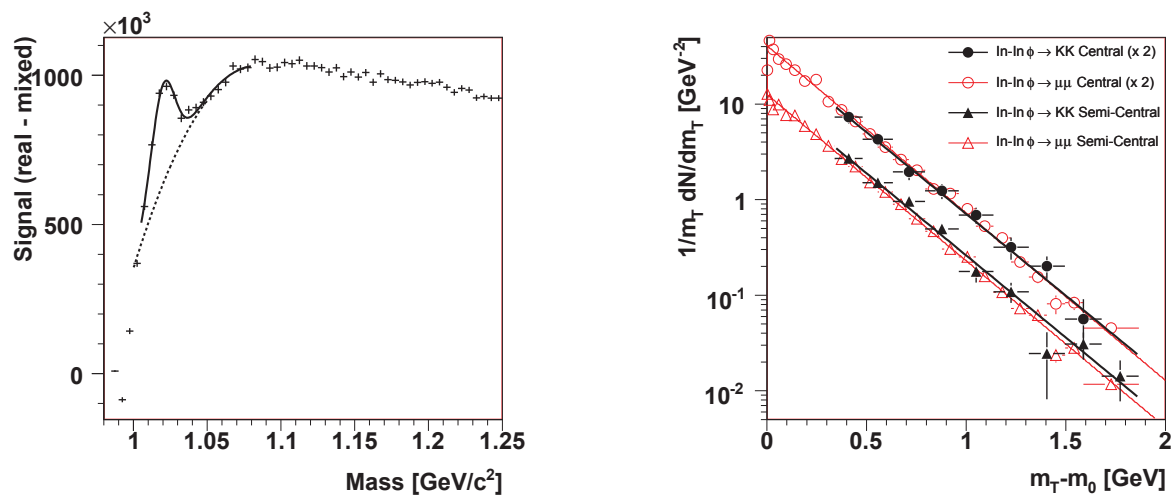

Fig. 2. Invariant mass spectrum of the opposite sign Fig. 3. Normalized transverse mass spectra for semikaon pairs after combinatorial background subtraction central (triangles) and central (circles) collisions for for $p_{T}>0.9 \mathrm{GeV} / c$ integrated in centrality. the $\phi \rightarrow K^{+} K^{-}$channel (full symbols) compared to the corresponding ones in muon pairs (open symbols) [28]. Results for central collisions are scaled by a factor 2 in order to improve readibility. Colour online.

The raw $\phi$ multiplicity is obtained measuring the number of $\phi$ mesons with $p_{T}>0.9 \mathrm{GeV} / c$, corrected for efficiency, acceptance and branching ratio in kaon pairs, divided by the total number of events selected for this analysis.

\subsection{Results}

Fig. 3 shows the transverse mass spectra for central and semi-central collisions in both the $\mu^{+} \mu^{-}$and $K^{+} K^{-}$channels. All the spectra are normalized to the $\phi$ multiplicity. The $m_{T}$ spectra are fitted with the function $1 / m_{T} d N / d m_{T} \propto e^{-m_{T} / T_{\text {eff }}}$. Results in the kaon channel are in good agreement with the ones obtained in dimuons in the full $p_{T}$ range, as reported in Table 1. Since in presence of radial flow the $T_{\text {eff }}$ value may depend on the $p_{T}$ range, the fit to the dimuon spectra was restricted to the range $p_{T}>0.9 \mathrm{GeV} / c$, giving $T_{\text {eff }}=252 \pm 4$ and $247 \pm 3 \mathrm{MeV}$ for semicentral and central collisions, respectively, still in agreement with the measurement in kaons.

The multiplicity per participant in the common range $p_{T}>0.9 \mathrm{GeV} / c$ is shown in Fig. 4 for both channels. The two data sets are in agreement within the uncertainties. A ratio between the $\phi$ yields in dimuons and in kaons larger than 1.18 in the common $p_{T}$ range is excluded at $95 \%$ C.L.

The $\phi$ multiplicity in the $K K$ decay channel was also calculated in the full $p_{T}$ range. Results are reported in the last column of Table 1 . The systematic error due to the uncertainty in the extrapolation to full $p_{T}$ caused by the error in the inverse slope was taken into account. It has to be stressed that the extrapolation to $p_{T}=0$ was done under the hypothesis that the inverse slope does not change. Models like AMPT [9] predict that kaon rescattering causes a depletion in the number of reconstructed 

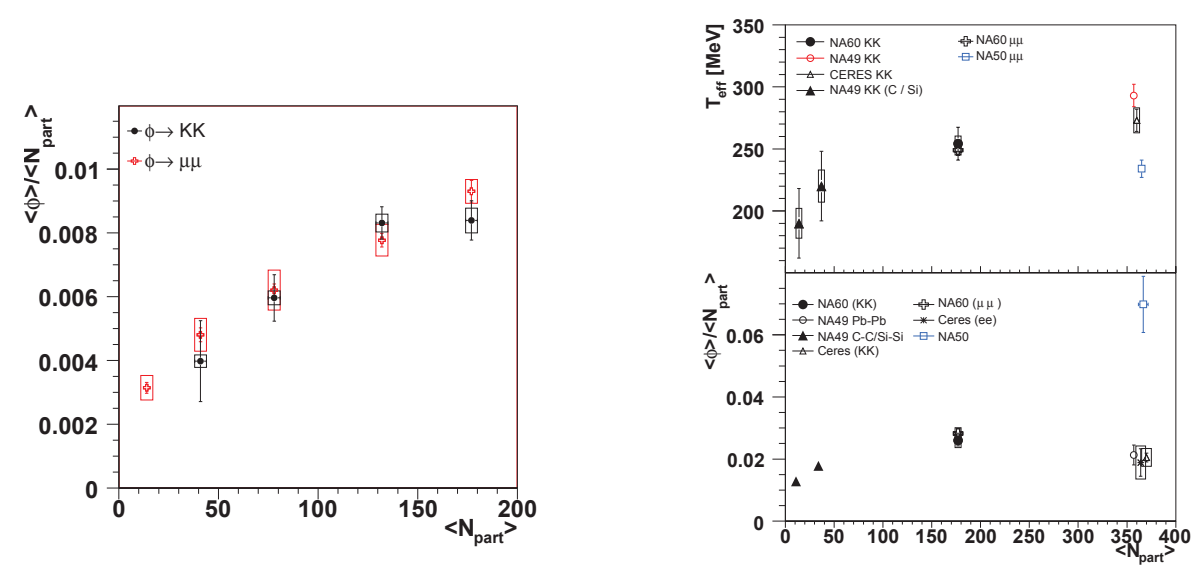

Fig. 4. $\langle\phi\rangle / N_{\text {part }}$ as a function of the number of partic- Fig. 5. Inverse slope (top) and $\langle\phi\rangle / N_{\text {part }}$ in full $p_{T}$ ipants in In-In collisions in the $\phi \rightarrow K^{+} K^{-}$(full cir- range (bottom) as a function of $N_{\text {part }}$ for central colcles) and $\phi \rightarrow \mu \mu$ (open crosses) channels for $p_{T}>$ lisions. Boxes: systematic errors. Colour online. $0.9 \mathrm{GeV} / c$. Boxes: systematic errors. Colour online.

$\phi \rightarrow K K$ which is concentrated at low $p_{T}$, causing an increase of $T_{\text {eff }}$ in that region. In $\mathrm{Pb}-\mathrm{Pb}$ central collisions, the effect would be already visible for $m_{T}-m_{0} \geq 0.34 \mathrm{GeV}$, corresponding to the region covered in this analysis. In that region, the difference between $T_{\text {eff }}$ in kaon and muon pairs would range from 30 to $50 \mathrm{MeV}$, and the fractional loss in kaons at $m_{T}-m_{0} \sim 0.34 \mathrm{GeV}$ would range from $35 \%$ to $50 \%$. Such effects are not seen in the NA60 spectra, suggesting that if any rescattering effect is present, it would be concentrated at lower $p_{T}$. In the range $0<m_{T}-m_{0}<0.34 \mathrm{GeV}$ the AMPT model predicts an inverse $m_{T}$ slope of about $330 \mathrm{MeV}$, close to the value observed by NA49 in central $\mathrm{Pb}-\mathrm{Pb}$ collisions. Since no theoretical predictions for In-In collisions are available, the effect of the change of slope in the extrapolation to $p_{T}=0$ was estimated under the extreme hypothesis $T_{\text {eff }}=330 \mathrm{MeV}$ for $0<m_{T}-m_{0}<0.34 \mathrm{GeV}$, while for $m_{T}-m_{0} \geq 0.34 \mathrm{GeV}$ the measured value is kept. This variation would cause a reduction of the $\phi$ yield in kaons of about $12 \%$. This quantity can be considered as a conservative estimation of the uncertainty in the extrapolation due to a possible suppression mechanism of the hadronic channel.

A comparison with other collision systems is done in Fig. 5, where the inverse slope $T_{\text {eff }}$ and the ratio $\langle\phi\rangle / N_{\text {part }}$ in the full $p_{T}$ range, are plotted in as a function of the number of participants for central $\mathrm{C}-\mathrm{C}, \mathrm{Si}-\mathrm{Si}$, In-In and $\mathrm{Pb}-\mathrm{Pb}$ collisions $[4,5]$. For this comparison, the systematic error on $N_{\text {part }}$, of about $5 \%$ in central collisions, is taken into account in the calculation of the ratio $\langle\phi\rangle / N_{\text {part }}$ for the NA60 points.

The inverse slope shows an initial fast increase at low $N_{\text {part }}$ values, that becomes less pronounced going towards higher $N_{\text {part }}$. A lower value is observed by NA50 as compared both to the CERES and NA49 measurements in $\mathrm{Pb}-\mathrm{Pb}$ and to the NA60 In-In points in the hadronic and dileptonic channels.

As stated above, in the presence of radial flow $T_{\text {eff }}$ depends on $p_{T}$. The NA60 analysis in dimuons [28], performed at low and high $p_{T}$ ranges, shows a difference limited to about $15 \mathrm{MeV}$ in In-In. This fact, complemented by the detailed blast wave analysis of the dimuon data discussed in [28], makes it difficult to ascribe only to radial flow the large differences in $T_{\text {eff }}$ shown in Fig. 5. A further flattening caused by kaon rescattering and absorption may lead to larger $T_{\text {eff }}$ values in the hadronic channel in $\mathrm{Pb}-\mathrm{Pb}$.

Concerning the enhancement, the NA60 measurements in the dilepton and hadron channels can differ up to $18 \%$ (at $95 \% \mathrm{CL}$ ) for $p_{T}>0.9 \mathrm{GeV} / c$ and $22 \%$ in full $p_{T}$ considering the uncertainty in the extrapolation arising from a possible suppression of the hadronic channel at low $p_{T}$. In Fig. 5 the NA50 result is extrapolated to full phase space using $T_{\text {eff }}=220 \mathrm{MeV}$, according to the NA50 measurement in peripheral collisions. Even assuming as an extreme case $T_{\text {eff }}=300 \mathrm{MeV}$, as obtained 

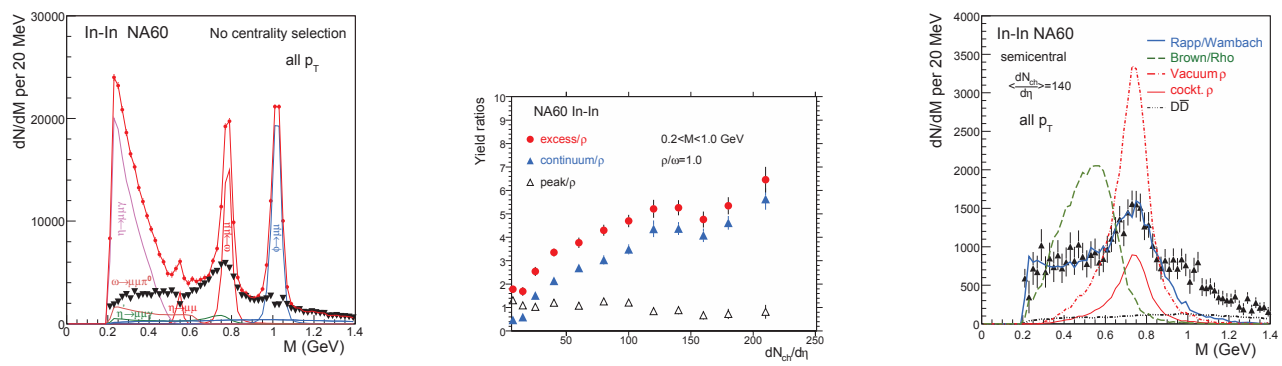

Fig. 6. Background-subtracted mass Fig. 7. Excess yield ratios for peak, Fig. 8. Excess dimuons compared spectrum before (dots) and after sub- continuum and total vs. centrality to theoretical predictions [17,16], traction of the known decay sources for the mass window $0.2<M<1 \mathrm{GeV}$. renormalized to the data in the mass (triangles).

Open charm is subtracted throughout. interval $M<0.9 \mathrm{GeV}$.

No acceptance correction applied.

by NA49 in central collisions, the NA50 enhancement would exceed by a factor of $\sim 2$ the central $\mathrm{Pb}-\mathrm{Pb}$ values measured in kaons.

The yield per participant measured in In-In exceeds the one observed in $\mathrm{Pb}-\mathrm{Pb}$ in the hadronic channel (both NA49 and CERES) by about 30\%. This might suggest a suppressing mechanism for the kaon channel, below experimental sensitivity in In-In, which shows up in $\mathrm{Pb}-\mathrm{Pb}$. The CERES measurement in dielectrons is in agreement with the one in kaons but, given the measurement errors, it cannot rule out differences of the order of 40-50\%, expected by models including kaon rescattering. If one assumes that no suppression mechanism affects the $\mathrm{Pb}-\mathrm{Pb}$ collisions, it is then difficult to understand why the $\phi$ multiplicity in central $\mathrm{Pb}-\mathrm{Pb}$ is smaller than in central In-In.

\section{$5 \rho$ spectral function and excess}

The background-subtracted dimuon mass spectrum in In-In collisions at $158 \mathrm{~A} \mathrm{GeV}$ integrated in centrality is shown in Fig. 6. The peripheral data can be completely described on the basis of the electromagnetic decays of neutral mesons [23], while in more central collisions a strong excess of muon pairs emerges in the low mass region. The high quality of NA60 data allows to isolate this excess with a priori unknown characteristics without any fits: the cocktail of the decay sources is subtracted from the total data using local criteria, which are solely based on the mass distribution itself. The $\rho$ is not subtracted. The excess resulting from this difference formation is illustrated in the same figure. It is characterized by a peak centred around the nominal $\rho$ pole and a continuum whose magnitude strongly depends on centrality. The peak and continuum yields are extracted counting the number of events in the excess in a $200 \mathrm{MeV}$ wide window centred around the $\rho$ pole mass $\left(N_{C}\right)$ and in two equally sided adjacent windows on the left and on the right of this one ( $N_{L}$ and $N_{R}$, respectively). The number of peak $\rho$ is $N_{C}-1 / 2\left(N_{L}+N_{R}\right)$, while the number of events in the continuum is $3 / 2\left(N_{L}+N_{R}\right)$. The dependence on centrality of peak and continuum is displayed in Fig. 7, where the yields are normalized to the (fictitious) cocktail $\rho$ under the assumption $\rho / \omega=1$, as in $p p$ collisions. The $\omega$ yield is found to be proportional to $d N_{c h} / d \eta$. While the peak $\rho$ slowly decreases with respect to the cocktail $\rho$, the continuum shows a very strong increase already for peripheral collisions. The excess $/ \rho$ ratio can directly be interpreted as the number of $\rho$ generations created by formation and decay during the fireball evolution, including freeze-out: the " $\rho$ clock", frequently discussed in the past. It reaches up to about 6 generations for central In-In collisions; selecting low $p_{T}$ this number doubles.

The excess and two main theoretical scenarios for the in-medium spectral properties of the $\rho$, broadening [17] and dropping mass [16], are shown in Fig 8. The model results are normalized to the data in the mass interval $M<0.9 \mathrm{GeV}$. The unmodified $\rho$, also shown in Fig. 8 (vacuum $\rho$ ), is clearly ruled out. The broadening scenario indeed gets close, while the dropping mass scenario can not describe the NA60 data. 

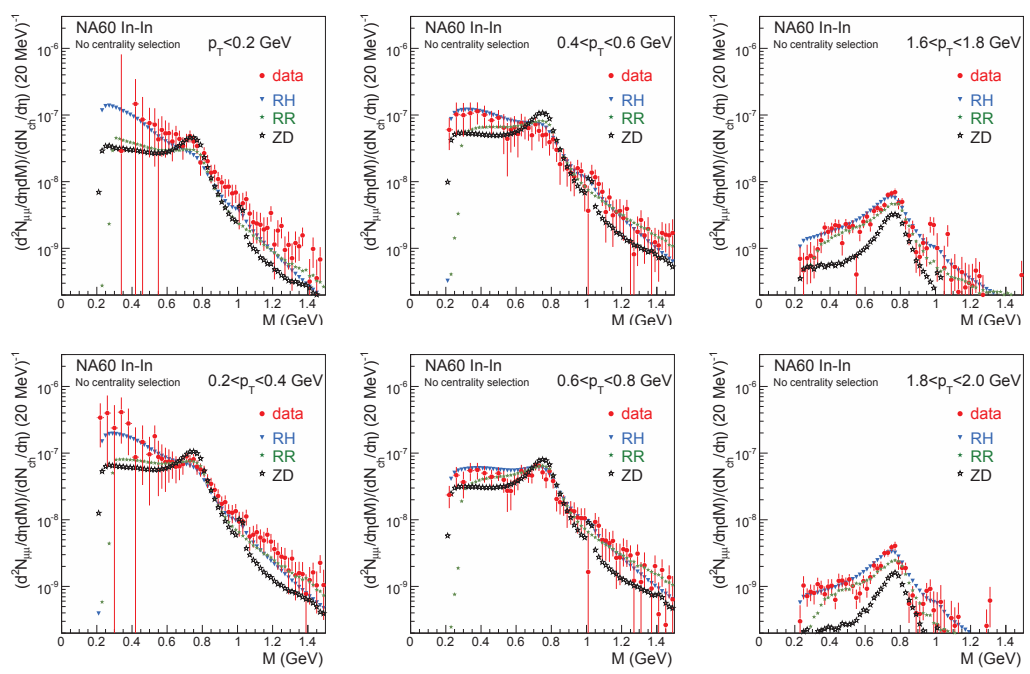

Fig. 9. Acceptance-corrected mass spectra of the excess dimuons in selected slices of $p_{T}$, with absolute normalization. The theoretical scenarios are labeled according to the authors RH [29], RR [30], and ZD [31]. In case of [29], the EoS- $\mathrm{B}^{+}$option is used, leading to a partonic fraction of about $65 \%$ in the IMR.

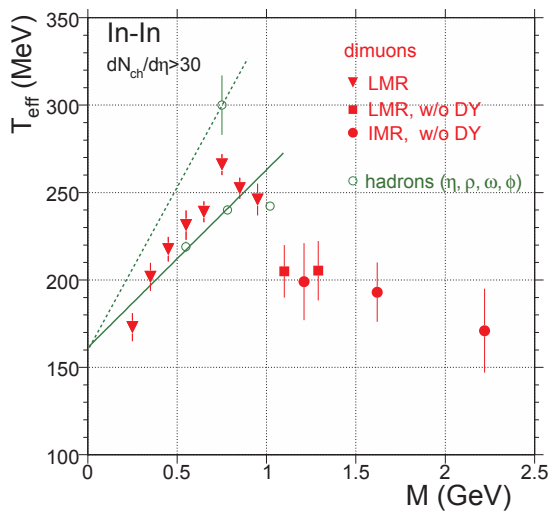

Fig. 10. Inverse slope parameter $T_{\text {eff }}$ vs. dimuon mass for the combined LMR/IMR regions of the excess in comparison to hadrons [24].

The acceptance-corrected mass spectra are shown, for several $p_{T}$ slices, in Fig. 9 (see [24,32, 33 ] for details on the acceptance correction). The mass spectra, absolutely normalized, are compared to several theoretical models $[34,35,30,29,31]$, calculated absolutely (not normalized to the data). Models are in good agreement with the data both in shapes and yields. At very low $p_{T}$, a strong rise towards low masses is seen in the data, reflecting the Boltzmann factor, i.e. the Plank-like radiation associated with a very broad, nearly flat spectral function. Only the Hees/Rapp scenario [29] is able to describe this part quantitatively, due to their particularly large contribution from baryonic interactions to the low-mass tail of the $\rho$ spectral function (as in [36]). At higher $p_{T}$, the influence of radial flow increasingly changes the spectral shapes, and at very high $p_{T}$ all spectra appear $\rho$-like.

The inverse slope parameters $T_{\text {eff }}$ extracted from exponential fits to the $m_{T}$ spectra in several mass windows, shown in [25], are plotted in Fig. 10 as a function of the dimuon mass, unifying the data from the low and intermediate mass regions. For $\mathrm{M}<1 \mathrm{GeV}$, a correction for Drell-Yan pairs is not done, due 
to their small contribution [24] and the intrinsic uncertainties at low masses [29]. In the extended LMR analysis up to $1.4 \mathrm{GeV}$, the 2 (square) points are corrected, as are all points of the IMR analysis [22]. In the region of overlap, the data are not statistically independent. The hadron data for $\eta, \omega$ and $\phi$ are extracted as a by-product of the cocktail subtraction procedure. They are also included in Fig. 10, as is the single value for the $\rho$-peak. Interpreting the latter as the freeze-out $\rho$ without in-medium effects, consistent with all present theoretical modeling [34,35,30,29,31], all four hadron values together with preliminary $\pi^{-}$data from NA60 can be subjected to a simple blast wave analysis [37]. This results in a reasonable set of freeze-out parameters of the fireball evolution and suggests the following consistent interpretation for the hadron and dimuon data together. Maximal radial flow is reached by the $\rho$, due to its maximal coupling to pions, while all other hadrons follow some hierarchy in earlier freeze-out. The $T_{\text {eff }}$ values of the dimuon excess rise nearly linearly with mass up to the pole position of the $\rho$, but stay always well below the $\rho$ line, completely consistent with the expectations for radial flow of an in-medium hadron-like source (here $\pi^{+} \pi^{-} \rightarrow \rho$ ) decaying continuously into lepton pairs.

For $M>1 \mathrm{GeV}$, the $T_{\text {eff }}$ values of the excess dimuons show a sudden decline by about $50 \mathrm{MeV}$ down to the IMR values. This decline is obviously connected to the in-medium emission itself, not to any peculiarities associated with the $\rho$ peak. Extrapolating the lower-mass trend set by a hadron-like source to beyond $1 \mathrm{GeV}$, such a fast transition is extremely hard to reconcile with emission sources which continue to be of dominantly hadronic origin in this region. A much more natural explanation would be a transition to a dominantly early, i.e. partonic emission source with processes like $q \bar{q} \rightarrow \mu^{+} \mu^{-}$, for which flow has not yet built up $[35,30]$. This observation is confirmed by the study of acceptance-corrected mass spectra, already described above. Assuming flat spectral functions, the yield integrated in momentum is $d N / d M \propto M^{3 / 2} \exp (-M / T)$, giving $T=205 \pm 12 \mathrm{MeV}$ when the mass spectrum is fitted in the region $1.2<M<2 \mathrm{GeV}$. Since $M$ is Lorentz-invariant, the mass spectrum is immune to any motion of the emitting sources, therefore $T$ is purely thermal. The observed value, considerably lower than $T_{c} \sim 170 \mathrm{MeV}$ thus directly implies partonic emission sources to dominate the intermediate mass region.

\section{References}

1. J. Rafelski, B. Muller, Phys. Rev. Lett. 48, 1066 (1982)

2. D. Lissauer, E.V. Shuryak, Phys. Lett. B253, 15 (1991)

3. F. Klingl, T. Waas, W. Weise, Phys. Lett. B431, 254 (1998)

4. V. Friese (NA49), Nucl. Phys. A698, 487 (2002)

5. C. Alt et al. (NA49), Phys. Rev. Lett. 94, 052301 (2005)

6. B. Alessandro et al. (NA50), Phys. Lett. B555, 147 (2003)

7. D. Jouan et al. (NA50), J. Phys.s G35, 104163+ (2008)

8. E.V. Shuryak, Nucl. Phys. A661, 119 (1999)

9. S. Pal, C.M. Ko, Z.w. Lin, Nucl. Phys. A707, 525 (2002)

10. E. Santini, G. Burau, A. Faessler, C. Fuchs, Eur. Phys. J. A28, 187 (2006)

11. S.C. Johnson, B.V. Jacak, A. Drees, Eur. Phys. J. C18, 645 (2001)

12. D. Adamova et al. (CERES), Phys. Rev. Lett. 96, 152301 (2006)

13. R. Arnaldi et al. (NA60 Collaboration), Eur.Phys.J. C64, 1 (2009), 0906.1102

14. R. Arnaldi et al. (NA60 Collaboration), Phys.Lett. B699, 325 (2011), 1104.4060

15. R.D. Pisarski, Phys.Lett. B110, 155 (1982)

16. G. Brown, M. Rho, Phys.Rept. 363, 85 (2002), hep-ph/0103102

17. R. Rapp, J. Wambach, Adv.Nucl.Phys. 25, 1 (2000), hep-ph/9909229

18. G. Agakichiev et al. (CERES Collaboration), Eur.Phys.J. C41, 475 (2005), nucl-ex/0506002

19. D. Adamova, G. Agakichiev, D. Antonczyk, H. Appelshauser, V. Belaga et al., Phys.Lett. B666, 425 (2008), nucl-ex/0611022

20. M. Abreu et al. (NA38 Collaboration, NA50 Collaborations), Eur.Phys.J. C14, 443 (2000)

21. A. Angelis et al. (HELIOS/3 Collaboration), Eur.Phys.J. C13, 433 (2000)

22. R. Arnaldi et al. (NA60 Collaboration), Eur.Phys.J. C59, 607 (2009), 0810. 3204

23. R. Arnaldi et al. (NA60 Collaboration), Phys.Rev.Lett. 96, 162302 (2006), nucl-ex/0605007 
24. R. Arnaldi et al. (NA60), Phys. Rev. Lett. 100, 022302 (2008), 0711. 1816

25. R. Arnaldi et al. (NA60 Collaboration), Eur.Phys.J. C61, 711 (2009), 0812 . 3053

26. M. Keil et al., Nucl. Instrum. Meth. A546, 448 (2005)

27. G. Usai et al. (NA60), Eur. Phys. J. C43, 415 (2005)

28. R. Arnaldi et al. (NA60), Eur. Phys. J. C64, 1 (2009), 0906.1102

29. H. van Hees, R. Rapp, Nucl. Phys. A806, 339 (2008), 0711 . 3444

30. T. Renk, J. Ruppert, Phys.Rev. C77, 024907 (2008), hep-ph/0612113

31. K. Dusling, D. Teaney, I. Zahed, Phys.Rev. C75, 024908 (2007), nucl-th/0604071

32. S. Damjanovic et al. (NA60 Collaboration), Nucl.Phys. A783, 327 (2007), nucl-ex/0701015

33. S. Damjanovic, Eur.Phys.J. C49, 235 (2007), nucl-ex/0609026

34. H. van Hees, R. Rapp, Phys.Rev.Lett. 97, 102301 (2006), hep-ph/0603084

35. J. Ruppert, C. Gale, T. Renk, P. Lichard, J.I. Kapusta, Phys.Rev.Lett. 100, 162301 (2008), Q706. 1934

36. E. Bratkovskaya, W. Cassing, O. Linnyk, Phys.Lett. B670, 428 (2009), 0805 . 3177

37. S. Damjanovic (NA60), J. Phys. G35, 104036 (2008), 0805.4153 